\title{
Memoria espacial a largo plazo en humanos entrenados en un laberinto virtual
}

\author{
David Luna ${ }^{1 *}$, Moisés Manzanares-Silva ${ }^{1}$, Katia Rodríguez-González $^{1,2}$ y Héctor López-Cruz ${ }^{1}$ \\ ${ }^{1}$ Instituto Politécnico Nacional, Centro Interdisciplinario de Ciencias de la Salud, Unidad Santo Tomás, México; ${ }^{2}$ Universidad Nacional \\ Autónoma de México, Facultad de Psicología, México
}

Recibido, marzo 17/2017

Concepto de evaluación, mayo 1/2017

Aceptado, junio 21/2017
Referencia: Luna, D., Manzanares-Silva, M., RodríguezGonzález, K. \& López-Cruz, H. (2018). Memoria espacial a largo plazo en humanos entrenados en un laberinto virtual. Acta colombiana de Psicología, 21(1), 70-82. doi: http:// www.dx.doi.org/10.14718/ACP.2018.21.1.4

\section{Resumen}

En este estudio se evaluó la memoria espacial a largo plazo en humanos. Para ello, se empleó un diseño cuasiexperimental en el que se entrenó a tres grupos de estudiantes de pregrado en un laberinto virtual de agua para localizar una plataforma oculta cuya ubicación era señalada por un conjunto de claves. Se realizó un pretest sin plataforma antes del entrenamiento, e inmediatamente después se condujo un postest (Grupo 0 h), así como después de un intervalo de retención de dos días (Grupo 48 h) y siete días (Grupo 168 h). En el pretest no se encontró evidencia de preferencia por alguna zona del laberinto. A lo largo de los ensayos de entrenamiento, el tiempo para encontrar la meta disminuyó sin diferencias entre grupos. Durante el postest, todos los grupos mostraron una preferencia por el cuadrante reforzado, sin embargo, el tiempo de permanencia, la distancia de nado y la precisión de la conducta de búsqueda en dicha zona fue equivalente entre el Grupo $0 \mathrm{~h}$ y el Grupo $48 \mathrm{~h}$, aunque mayor a la mostrada por el Grupo $168 \mathrm{~h}$. Estos datos indican cambios ocurridos $48 \mathrm{~h}$ después de la adquisición en la memoria espacial a largo plazo en humanos. Se discuten los resultados a partir de procesos generales de memoria y procesos específicos propuestos por teorías particulares de memoria espacial; y al final se abordan las implicaciones clínicas y pertinentes al campo de la psicología comparada.

Palabras clave: Laberinto virtual de agua, retención, olvido espontáneo, memoria espacial.

\section{Long-term spatial memory in humans trained in a virtual maze}

\begin{abstract}
In this study we evaluated the long-term spatial memory in humans. A quasiexperimental design was used in which three groups of undergraduate students were trained in a virtual water maze to locate a hidden platform whose location was indicated by a set of cues. A pre-test without platform was performed prior to the training, and a post-test was conducted immediately after this (Group 0h), or after a retention interval of two (Group 48h) or seven days (Group 168h). For the pre-test, there was no evidence of preference for any area of the maze. Throughout the training trials, the time to find the goal decreased without differences between groups. During the post-test, all groups showed a preference for the reinforced quadrant, although the spent time, swimming distance, and accuracy of the search behavior in that area was equivalent between Group $0 \mathrm{~h}$ and Group $48 \mathrm{~h}$, but higher than that shown by the Group $168 \mathrm{~h}$. These data indicate changes in long-term spatial memory in humans, occurring after an interval of $48 \mathrm{~h}$ after its acquisition. The results are discussed on the basis of general memory processes and specific processes proposed by particular spatial memory theories. The clinical and comparative psychology implications are also addressed.

Key words: Virtual water maze, retention, spontaneous forgetting, spatial memory.
\end{abstract}

\footnotetext{
* Av. de los Maestros s/n, Miguel Hidalgo, Santo Tomas, 11340, Ciudad de México.+52 01555729 6000, ext. 63414. dglunap@ipn.mx
} Este estudio fue financiado por el Instituto Politécnico Nacional a través del apoyo SIP20160878 otorgado al primer autor. 


\title{
Memória espacial de longo prazo em humanos treinados num labirinto virtual
}

\author{
Resumo
}

\begin{abstract}
Neste estudo, avaliou-se a memória espacial de longo prazo em humanos. Para isso, empregou-se um desenho quaseexperimental no qual se treinou três grupos de estudantes de graduação num labirinto virtual de água para localizar uma plataforma oculta cuja posição era sinalizada por um conjunto de chaves. Realizou-se um pré-teste sem plataforma antes do treinamento, e imediatamente depois se conduziu um pós-teste (Grupo $0 \mathrm{~h}$ ), assim como depois de um intervalo de retenção de dois dias (Grupo 48 h) e de sete dias (Grupo 168 h). No pré-teste, não se encontrou evidência de preferência por alguma área do labirinto. Ao longo dos ensaios de treinamento, o tempo para encontrar a meta diminuiu sem diferenças entre grupos. Durante o pós-teste, todos os grupos mostraram uma preferência pelo quadrante reforçado, contudo o tempo de permanência, a distância de nado e a precisão do comportamento de busca nessa área foi equivalente entre o Grupo $0 \mathrm{~h}$ e o Grupo $48 \mathrm{~h}$, embora maior à amostragem pelo Grupo $168 \mathrm{~h}$. Esses dados indicam mudanças ocorridas $48 \mathrm{~h}$ depois da aquisição na memória espacial de longo prazo em humanos. Discutem-se os resultados a partir de processos gerais de memória e de processos específicos propostos por teorias particulares de memória espacial; ao final, abordam-se as implicações clínicas e pertinentes ao campo da psicologia comparada.

Palavras-chave: Esquecimento espontâneo, labirinto virtual de água, memória espacial, retenção.
\end{abstract}

\section{INTRODUCCIÓN}

Mediante la memoria espacial, los organismos pueden adquirir, retener y recuperar conocimiento acerca de las características del entorno y así trazar rutas directas entre ellos y recordar la ubicación de metas útiles para resolver diversos problemas adaptativos (Postma, Jager, Kessels, Koppeschaar \& Van Honk, 2004). Como en otros tipos de memoria, en el establecimiento de la memoria espacial participan procesos de adquisición, consolidación y recuperación de información, en los cuales el hipocampo (HC) juega un papel crucial (Florian \& Roullet, 2004; GoodrichHunsaker, Livingstone, Skelton \& Hopkins, 2009; Moser \& Moser, 1998).

Desde el enfoque de los sistemas de memoria (Squire, 2004), la memoria espacial ha sido considerada como parte de la memoria declarativa -ya sea como un componente de la memoria episódica (Morellini, 2013) o como un subsistema independiente de esta (Morris, 2013) - y su función ha sido la de registrar el contexto espacial de los eventos experimentados por el organismo (Burgess, Maguire \& O'Keefe, 2002). Además, dado que la memoria declarativa se incluye en la memoria a largo plazo, dicha pertenencia es extensible a la memoria espacial (Demas, Nelson, Krueger \& Yarowsky, 1996; Morris, 2013).

El concepto de memoria a largo plazo se ha definido de diversas formas (Morris \& Mayes, 2004), aunque un criterio de temporalidad indica que la retención de la información contenida en esta es de al menos $24 \mathrm{~h}$ (Izquierdo, Medina, Vianna, Izquierdo \& Barros, 1999), pudiendo extenderse de semanas a meses (Roth, LaDage \& Pravosudov, 2012), incluso hasta años (Ruetti, Justel \& Bentosela, 2009). Bajo estos supuestos, la información espacial retenida por más de $24 \mathrm{~h}$ puede ser considerada memoria espacial a largo plazo.

Evidencia de este tipo de memoria se ha obtenido con animales no humanos, por ejemplo, aves (Mettke-Hofmann \& Gwinner, 2003; Roth et al., 2012; Shiflett, Tomaszycki, Rankin \& DeVoogd, 2004; Wilkie \& Willson, 1989) y roedores (Carmack, Block, Howell \& Anagnostaras, 2014; Clark, Broadbent \& Squire, 2005; Conejo, Cimadevilla, González-Pardo, Méndez-Couz \& Arias, 2013; Demas et al., 1996; Méndez-Couz, Conejo, González-Pardo \& Arias, 2015). En estos estudios se interpuso un intervalo de retención (IR) entre el entrenamiento en una tarea espacial y una prueba, cuya duración estuvo en el rango de días, semanas o meses.

Los datos obtenidos en estos trabajos han demostrado que las aves retienen la información sobre la ubicación de una meta hasta por seis meses después de su adquisición, sin una pérdida significativa de información debida al paso del tiempo (Roth et al., 2012). En cambio, se ha encontrado que los roedores pierden información gradualmente y con variaciones de acuerdo a la tarea empleada, en función del aumento en la duración del IR (Clark et al., 2005). A pesar de lo anterior, tanto en aves como en roedores se ha confirmado la participación del $\mathrm{HC}$ en el establecimiento de la memoria espacial a largo plazo (Clark et al., 2005; Shiflett et al., 2004).

Por otra parte, aunque la memoria espacial ha sido ampliamente investigada en humanos expuestos a entornos virtuales y reales (Chamizo, Aznar-Casanova \& Artigas, 2003; Goodrich-Hunsaker et al., 2009; Hardt, Hupbach \& 
Nadel, 2009; Jacobs, Laurance \& Thomas, 1997; Schoenfeld, Foreman \& Leplow, 2014; Spieker, Astur, West, Griego \& Rowland, 2012; Ross, Skelton \& Mueller, 2006), los estudios que abordan el efecto del paso del tiempo en la retención de la memoria espacial son actualmente escasos, y la mayoría de ellos han sido conducidos como diseños de caso único que analizan la memoria espacial de pacientes con daño en la cara medial del lóbulo temporal, incluyendo el HC.

El resultado general de dichos trabajos es la ausencia de retención a largo plazo de la memoria espacial adquirida en diversas tareas espaciales (Maguire, Nannery \& Spiers, 2006; Rosenbaum, Cassidy \& Herdman, 2015; Rosenbaum, Winocur, Grady, Ziegler \& Moscovitch, 2007; Teng \& Squire, 1999; Tramoni et al., 2011). Sin embargo, uno de estos estudios reportó que la población sana retiene la información espacial durante varias semanas después de su adquisición (Tramoni et al., 2011). Si bien las investigaciones con animales no humanos y algunas con humanos son consistentes con el concepto de memoria espacial a largo plazo, la diversidad de tareas espaciales e IR utilizados para las distintas especies estudiadas complica el análisis sistemático, lo que a su vez que dificulta su desarrollo teórico (Moscovitch, Nadel, Winocur, Gilboa \& Rosenbaum, 2006; Rosenbaum et al., 2007).

La importancia de la investigación sistemática de la memoria espacial a largo plazo está dada por sus implicaciones tanto teóricas como aplicadas. Para el primer caso, a pesar de que la teoría del mapa cognitivo (TMC) (O'Keefe \& Nadel, 1978; Manns \& Eichenbaum, 2009) es considerada una de las aproximaciones más influyentes en la investigación en memoria espacial (Kelly \& Gibson, 2007), sus planteamientos sobre el efecto del paso del tiempo en este tipo de memoria han sido poco desarrollados e investigados. Una de las tesis de la TMC es que el paso del tiempo no tiene efecto sobre la memoria espacial; en consecuencia, esta teoría no predice diferencias en la memoria espacial cuando se evalúa en distintos IR posteriores a su adquisición. $\mathrm{Si}$ bien los datos obtenidos con aves son consistentes con esa predicción, los reportados con roedores no lo son, y para los humanos los datos actuales son insuficientes para poder verificar o refutar tal planteamiento.

Ahora bien, las implicaciones en un contexto aplicado se vinculan con el establecimiento de modelos experimentales para la investigación clínica en memoria, y existe evidencia de un déficit en la memoria episódica producto del envejecimiento, siendo una de sus expresiones la dificultad para recuperar información acerca del contexto de ocurrencia del evento meta (Chalfonte \& Johnson, 1996), siendo parte de esa información proporcionada por la memoria espacial (Burgess et al., 2002). Así, validar una metodología para el estudio de la memoria espacial a largo plazo en humanos sería de utilidad para describir y comparar sus características en poblaciones sanas y en poblaciones clínicas, lo que sería también una potencial herramienta de diagnóstico e incluso para la evaluación de tratamientos farmacológicos (Schoenfeld et al. 2014; Spieker et al., 2012).

El laberinto de agua de Morris (1984) es la tarea espacial más utilizada en la investigación de la memoria espacial con roedores (D'Hooge \& De Deyn, 2001; Vorhees \& Williams, 2014), y, empleando su versión virtual, es posiblemente el instrumento más utilizado con humanos, (Jacobs et al., 1997). Adicionalmente, la evidencia de semejanzas neurobiológicas y de ejecución, que es además dependiente del $\mathrm{HC}$ en ambas especies (Goodrich-Hunsaker et al., 2009; Morris, Garrud, Rawlins \& O'Keefe, 1982; Wooley et al., 2013), facilita la comparación de los resultados obtenidos en diversos estudios.

En esta tarea, el sujeto debe nadar en una alberca hasta aprender la ubicación de una plataforma oculta debajo del nivel del agua. Posteriormente, durante un ensayo de prueba sin plataforma, los sujetos muestran típicamente una preferencia por el área en que esta se localizó. En general, en roedores los ensayos de entrenamiento se distribuyen en diversas sesiones diarias y la prueba se realiza después del ensayo final o tras un IR de 24 h (Morris, 1984; Vorhees \& Williams, 2014), mientras que en humanos se realiza tanto el entrenamiento como la prueba en una sola sesión (Astur, Ortiz \& Sutherland, 1998; Goodrich-Hunsaker et al., 2009; Hamilton, Driscoll \& Sutherland, 2002; Jacobs et al., 1997; Kolarik et al., 2016; Schoenfeld et al., 2014).

Además, el momento en que se realice la prueba tiene implicaciones teóricas dado el tipo de memoria evaluada, pues se ha señalado que su conducción inmediatamente al término del entrenamiento requiere del uso de la memoria espacial a corto plazo, mientras que su conducción demorada $(\geq 24 \mathrm{~h}$ ) requiere de la memoria espacial a largo plazo (Baldi, Efoudebe, Lorenzini \& Bucherelli, 2005; Spreng, Rossier \& Schenk, 2002; Vorhees \& Williams, 2014).

Para verificar el contenido de ambas memorias, en un estudio (Baldi et al., 2005) se entrenó a ratas en el laberinto de agua durante sesiones diarias, y en cada sesión se realizó un pre y un postest. En todas las sesiones, el postest reveló la preferencia por el cuadrante reforzado; en cambio, para el pretest, el mismo resultado ocurrió solo en las últimas sesiones. Los autores argumentaron el uso de una memoria espacial a corto plazo durante el postest; mientras que para resolver el pretest fue requerida una memoria espacial a largo plazo, establecida solo cuando había avanzado el entrenamiento. Estos resultados indican que la ejecución en una prueba inmediata no es un indicador fiable de la memoria espacial a largo plazo, al menos para roedores. Actualmente no se encuentran estudios semejantes con 
humanos, lo que impide evaluar semejanzas y diferencias entre su memoria espacial a corto y a largo plazo.

Teniendo en cuenta lo anterior, el objetivo del presente estudio fue describir el efecto del paso del tiempo en la memoria espacial en humanos. Así, después del entrenamiento en un laberinto virtual de agua (LVA), un grupo de participantes realizó un ensayo de prueba inmediata, mientras que otros dos grupos lo realizaron en forma demorada (48 y $168 \mathrm{~h}$, respectivamente). El supuesto subyacente a este diseño es que la prueba inmediata revelará el contenido de la memoria espacial a corto plazo, mientras que las pruebas demoradas revelarán el de la memoria espacial a largo plazo.

\section{MÉTODO}

\section{Participantes}

Se empleó una técnica de muestreo por conveniencia para reclutar a 36 estudiantes de pregrado, de ambos sexos y en un rango de edad de 18 a 16 años de edad $(\mathrm{M}=20.69$, $\mathrm{DE}=1.99)$, los cuales fueron divididos en tres grupos igualados para la variable sexo (Grupo $0 \mathrm{~h}$, Grupo $48 \mathrm{~h}$, y Grupo $168 \mathrm{~h}$ ), con un total de siete mujeres y cinco hombres cada grupo. Los participantes desconocían los objetivos del experimento y no tenían experiencia en la tarea empleada ni en alguna semejante; además, contaban con un buen estado de salud general, vista normal o corregida, y no consumían ningún fármaco o sustancia que afectara su desempeño cognitivo o motor.

El desempeño en el experimento (es decir, el aprendizaje de la tarea experimental) no justificó la exclusión de los datos de ningún participante. La colaboración fue voluntaria y previa la realización del estudio, a todos los participantes se les informó sobre sus derechos, de acuerdo a la Declaración de Helsinski (World Medical Association Declaration of Helsinki, 2008). Adicionalmente, en la conducción del estudio se siguió la normativa y consideraciones éticas para la investigación vigentes actualmente en México (Sociedad Mexicana de Psicología, 2010).

\section{Aparatos y materiales}

El experimento se realizó en un cubículo amueblado con una silla y un escritorio sobre el cual se colocó una computadora laptop de 14 pulgadas - cargada con Windows 7 y un monitor a color ajustado a una resolución de 1024 x 768 pixeles, en la cual se presentó la tarea experimental.

\section{Tarea experimental}

La tarea experimental fue diseñada con el programa Maze Suite v. 2.3.0.1 (Ayaz et al., 2011) y consistió en un LVA en el que se presentó -en perspectiva de primera personauna alberca octagonal con agua azul claro rodeada por un muro gris sobre el cual se colocaron, en forma distribuida, diversas figuras geométricas tridimensionales (E: esfera; C: cubo; PR: paralelepípedo rectangular) de diferentes colores (Am: amarillo; Az: azul; V: verde; R: rojo). La alberca se ubicó en una habitación con muros de colores y texturas diferentes, y con un cielo nublado a manera de plafón. Los elementos de la habitación y las figuras geométricas tridimensionales fungieron como claves útiles para localizar una plataforma oculta debajo del nivel del agua, cuyo tamaño fue de aproximadamente el $3.5 \%$ del área total de
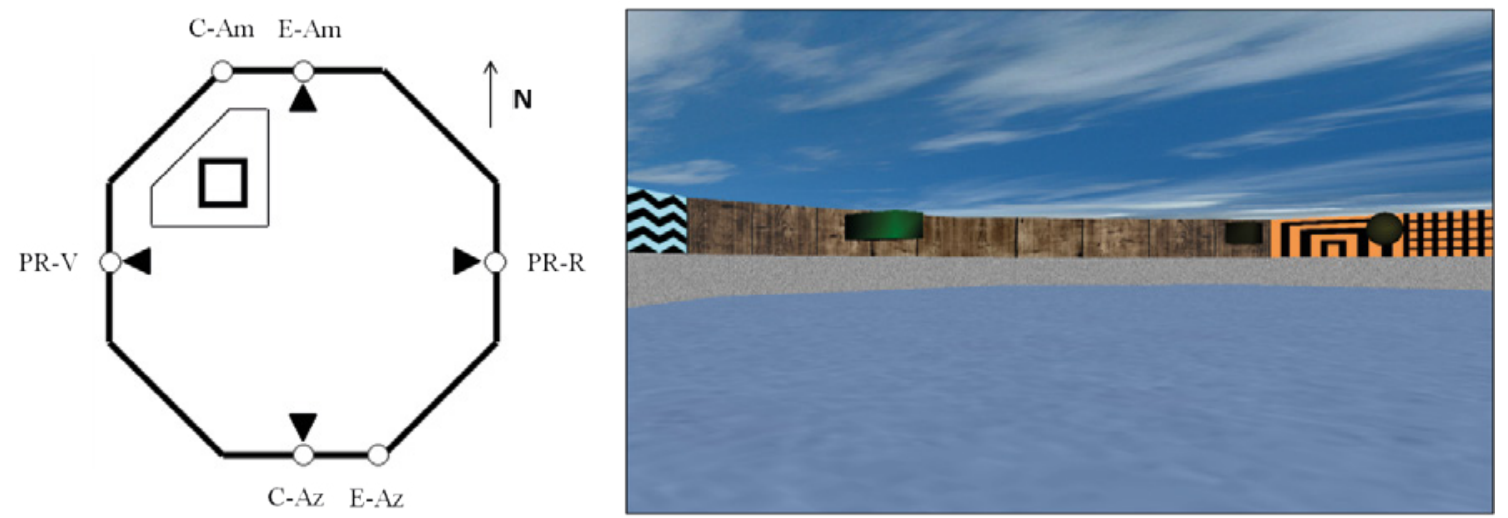

Figura 1. Izquierda: Vista esquemática del LVA. El recuadro interno indica la ubicación de la plataforma oculta (línea gruesa) y el subcuadrante interno (línea delgada). Las flechas al centro de los muros son los puntos de partida y las letras en la leyenda junto a cada círculo abierto señalan la figura geométrica tridimensional empleada y su color. Derecha: Vista del LVA desde la perspectiva del participante 
la alberca (véase Figura 1). El desplazamiento en el LVA fue controlado con las flechas de dirección en el teclado de la computadora: $(\uparrow)$ nado adelante $y(\downarrow)$ nado atrás para el movimiento, y $(\leftarrow)$ vuelta a la izquierda y $\rightarrow$ ) vuelta a la derecha para la rotación sobre el eje del participante (Hardt et al., 2009). Un giro de $360^{\circ}$ tomó $5 \mathrm{~s}$, aproximadamente, mientras que cruzar la alberca requirió alrededor de $7 \mathrm{~s}$.

\section{Diseño}

Se trató de un diseño cuasiexperimental con varios grupos de comparación y mediciones de pretest y postest. Los grupos fueron igualados para la variable sexo. Tras un pretest, todos los grupos fueron expuestos a 12 ensayos de entrenamiento en la tarea y, posteriormente, se realizó el postest. La variable a manipular fue el IR antes de realizar el postest.

\section{Procedimiento}

El experimento se realizó en forma individual y comprendió cinco fases: (a) práctica, (b) preentrenamiento, (c) pretest, (d) entrenamiento, y (e) postest. La fase de práctica fue útil para familiarizar a los participantes con el desplazamiento en el LVA, y junto con la fase de preentrenamiento permitieron evaluar su habilidad motora, motivacional y visual (Hamilton, Kodituwakku, Sutherland $\&$ Savage, 2003). En estas fases se omitió la presentación de los muros de la habitación virtual, el plafón y las claves.

El Grupo 0 h realizó las cinco fases en una sola sesión de alrededor de $15 \mathrm{~min}$, mientras que el Grupo $48 \mathrm{~h} \mathrm{y} \mathrm{el}$ Grupo $168 \mathrm{~h}$ realizaron las cuatro primeras fases en una sesión y la última fase en una sesión posterior. Para cada fase, al término de un ensayo los participantes debían presionar la tecla ENTER en el teclado de la computadora con el fin de presentar el evento siguiente.

La fase de práctica comprendió un ensayo de $30 \mathrm{~s}$ sin plataforma, cuyo inicio fue al centro del LVA, con la vista orientada hacia el norte. Se instruyó a los participantes para imaginar que se encontraban nadando en una alberca y entonces se les solicitó desplazarse libremente en ella. Posteriormente, se realizó una fase de preentrenamiento con dos ensayos, que fue útil para enseñar a los participantes a localizar una plataforma visible en el LVA. Después, se les solicitó desplazarse en el entorno hasta hacer contacto con una plataforma visible sobre el nivel del agua y ubicada en una zona de la alberca.

Cada ensayo de esta fase comenzó al centro del cuadrante noreste del LVA y se presentó una plataforma visible sobre el nivel del agua ubicada frente a la parte central del muro norte (primer ensayo) o del muro sur (segundo ensayo). El ensayo terminó cuando el participante hacía contacto con la plataforma y aparecía al centro del monitor una leyenda que decía "Aquí está la plataforma" (retroalimentación positiva). Concluida esta fase, se indicó a los participantes que en los ensayos posteriores la plataforma estaría oculta debajo del nivel del agua y que, para evitar ahogarse, debían encontrarla antes de que transcurriera un límite de tiempo.

Se les mencionó también que diversos elementos del entorno podrían ayudarles a determinar la posición de la plataforma. Tras aclarar las dudas pertinentes, el investigador abandonó el ambiente experimental y el participante pudo comenzar con el pretest -fase que comprendió un ensayo de $60 \mathrm{~s}$ sin plataforma-; el participante inició el ensayo al frente del muro sur de la alberca y, dada la ausencia de la plataforma, a su término se presentó un mensaje en el centro del monitor que decía "Has muerto" (retroalimentación negativa).

Inmediatamente después empezó la fase de entrenamiento, que incluyó 12 ensayos con una duración máxima de $60 \mathrm{~s}$ con la plataforma oculta presente y ubicada en el cuadrante Noroeste. Cada ensayo comenzó en un punto cardinal distinto - programado semialeatoriamente para no presentar el mismo en forma consecutiva- $\mathrm{y}$ con la vista del participante orientada hacia el muro del LVA. En esta fase, si el participante localizaba la plataforma, se mostraba la retroalimentación positiva; y en caso de agotar la duración del ensayo sin encontrarla, se presentaba la retroalimentación negativa. Después de que se daba la retroalimentación pertinente, se programaba el ensayo o fase siguiente según el caso. Finalmente, se condujo un postest con las mismas características que el pretest.

El Grupo $0 \mathrm{~h}$ realizó el postest inmediatamente después de la fase de entrenamiento; en cambio, los participantes del Grupo 48 h y del Grupo 168 h fueron citados por el investigador para completar el experimento dos y siete días después, respectivamente. Durante la sesión final, los participantes de estos do grupos fueron sentados frente a la computadora, cuyo monitor mostraba la leyenda "Por favor localiza la plataforma oculta tal y como lo hiciste antes". El postest comenzó tras presionar la tecla ENTER en el teclado de la computadora. Después de esta fase, el experimento concluyó para cada uno de los grupos.

\section{Indicadores conductuales}

Se registraron los siguientes indicadores conductuales:

1. Latencia de escape: tiempo transcurrido desde el inicio del ensayo hasta localizar la plataforma oculta.

2. Permanencia en cuadrante reforzado: tiempo transcurrido en el cuadrante del LVA en que se localizó la plataforma oculta. Aunque esta es la medida más comúnmente analizada en estudios que emplean el laberinto de agua, su validez 
ha sido cuestionada (Hardt et al., 2009), por lo que se ha sugerido emplear áreas de análisis ad hoc, generalmente de menor tamaño, para revelar con más detalle la calidad de la memoria espacial (es decir, los subcuadrantes) (Blokland, Geraerts \& Been, 2004).

3. Subcuadrante interno: un área periférica a la ubicación de la plataforma, que correspondió al $37 \%$ del área total del cuadrante reforzado, y que excluyó una banda adyacente al perímetro de este (véase Figura 1).

4. Índice de precisión (IP): proporción del tiempo permanecido en el subcuadrante interno en relación con el tiempo transcurrido en el cuadrante reforzado. Se obtiene al dividir el tiempo de permanencia en el subcuadrante entre la permanencia en el cuadrante reforzado. El resultado tiene un rango de 0 a 1 , e indica la zona donde preferentemente se buscó la plataforma: un IP $>.5$ refleja una mayor búsqueda en el subcuadrante interno (es decir, mayor precisión); un IP $<.5$ refleja mayor búsqueda en la zona periférica del cuadrante reforzado (es decir, menor precisión); y un IP $=.5$ refleja que la búsqueda es indistinta entre estas dos áreas. $\mathrm{Su}$ uso atiende a la necesidad de evaluar la precisión en la conducta de búsqueda en tareas espaciales más que la permanencia en zonas específicas (Kolarik et al., 2016).

5. Distancia de nado en el LVA y en el cuadrante reforzado: longitud en unidades virtuales $(u v)$ del trayecto recorrido al buscar la plataforma oculta.

De forma parecida a lo reportado en otros estudios (Astur, Purton, Zaniewski, Cimadevilla \& Markus, 2016; Kolarik et al., 2016), estas variables se consideraron como un indicador del nivel motivacional de los participantes durante la prueba. El indicador 1 se empleó en la fase de entre-namiento, mientras que el 2, el 3 y el 5 se utilizaron en ambas pruebas, y el 4 solo en el postest.

\section{Análisis de datos}

El análisis de los datos se realizó con el programa SPSS v. 20. Inicialmente, se evaluó la normalidad de los datos mediante la prueba Shapiro-Wilk, y los análisis posteriores incluyeron pruebas $t$ de una cola para un grupo y análisis de varianza (ANOVA) con la prueba post hoc de la Diferencia Honestamente Significativa de Tukey (DHS). Estas dos pruebas son robustas, de forma que garantizan la certeza de sus resultados, incluso con desviaciones al supuesto de normalidad, tal como se presentó en algunas de las variables analizadas en este estudio, aunque debe tenerse precaución con las inferencias realizadas. Ambas pruebas se consideran como la mejor opción para el análisis de datos, incluso con grupos de $\mathrm{n}<5$ frente a pruebas no paramétricas (Khan \& Rayner, 2003; De Winter, 2013).
También se calculó la $d$ de Cohen (pruebas $t$ ) y la eta cuadrada parcial ( $\eta_{p}^{2}$; ANOVA) como índices del tamaño del efecto, considerando un efecto pequeño, mediano y grande a una $d \geq .20, .50, \mathrm{y} .80$, y a una $\eta_{p}{ }^{2} \geq .01, .06, .14$, respectivamente (Aron \& Aron, 2001). En todas las pruebas se consideró un resultado como significativo cuando el valor de $p$ era menor o igual a .05 ( $p \leq .05)$.

\section{RESULTADOS}

Inicialmente, en esta sección se presentan los resultados sobre la normalidad de los datos y la equivalencia inicial de los grupos durante la fase de práctica y la fase de preentrenamiento. Posteriormente, se presentan los resultados obtenidos durante el entrenamiento; $\mathrm{y}$ al finalizar, se comparan los obtenidos durante el pretest y el postest.

Normalidad de los datos y equivalencia inicial de los grupos

La prueba Shapiro-Wilk arrojó evidencia de la normalidad de los datos $(p>.05)$ de todos los grupos para las variables de permanencia en cuadrante reforzado, distancia de nado en el LVA y en el cuadrante reforzado, e IP. Para la permanencia en el subcuadrante interno, esta distribución se demostró en el Grupo $48 \mathrm{~h}$ en el pretest, y en los grupos $0 \mathrm{~h}$ y $168 \mathrm{~h}$ en el postest. Para el entrenamiento, un mayor número de ensayos por grupo no cumplió el requisito de normalidad $(p<.05)$. A pesar de lo anterior, se procedió con el análisis paramétrico antes descrito, dadas las ventajas de este sobre análisis no paramétricos, incluso bajo las condiciones reportadas. Adicionalmente, el análisis preliminar no mostró diferencias entre grupos en la distancia de nado en el LVA durante la fase de práctica y de preentrenamiento, ni en la latencia para alcanzar la plataforma visible cuando los ensayos de esta última fase fueron colapsados en un solo bloque $(p>.05)$.

\section{Ejecución durante el entrenamiento}

La Figura 2 muestra una disminución sistemática y sin diferencias entre grupos para la latencia de escape, lo cual se confirmó al conducir un ANOVA 3 vs. 2 con los factores de grup os ( 0 h vs. 48 h vs. 168 h) y ensayos (1-12). Este análisis reveló un efecto principal para el factor ensayos $F_{(11,363)}=26.13, p<.01, \eta_{p}{ }^{2}=.44$, siendo la ejecución en el Ensayo 1 distinta a la del Ensayo $8(p<.01)$. 


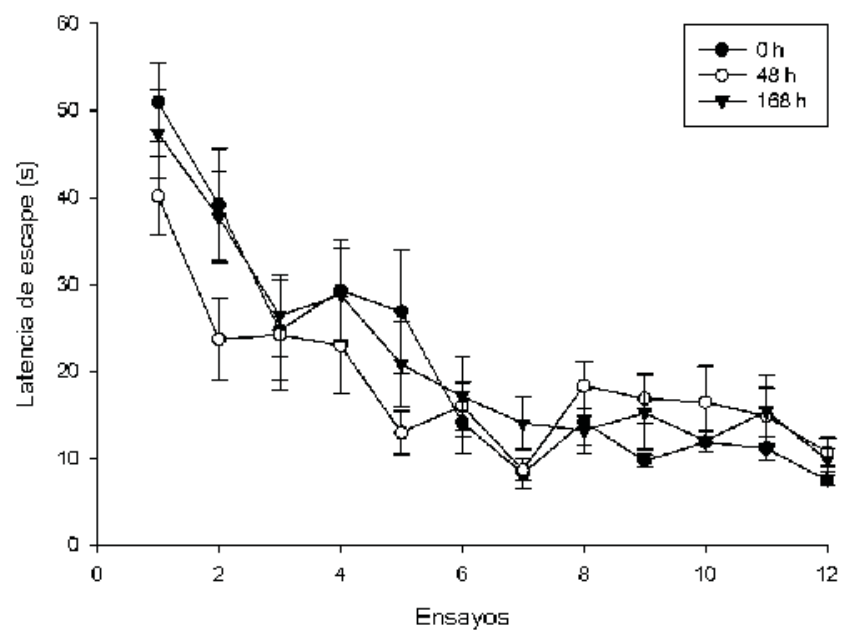

Figura 2. Latencia de escape durante la fase de entrenamiento.

Comparación de la ejecución de los grupos durante el pretest y el postest

Para todos los grupos, la permanencia en el cuadrante reforzado fue menor durante el pretest respecto de registrada para el postest (véase Figura 3). El ANOVA de tres grupos ( $0 \mathrm{~h}$ vs. $48 \mathrm{~h}$ vs. $168 \mathrm{~h}$ ) vs. dos pruebas (pretest vs. postest) reveló un efecto principal para cada factor y su interacción, a decir: (a) grupos: $F_{(2.66)}=3.74, p<.01$, $\eta_{p}^{2}=.10$; (b) pruebas: $F_{(1.66)}=53.35, p<.01, \eta_{p}^{2}=.44 ; \mathrm{y}$ (c) grupos vs. pruebas: $F_{(2.66)}=3.29, p<.05, \eta_{p}{ }^{2}=.09$. Esta interacción se analizó de dos maneras, cada una empleando un ANOVA unifactorial.

En el primer análisis se comparó entre los grupos el desempeño durante cada prueba, donde no se detectaron diferencias para el pretest $(p>.05)$, aunque sí las hubo para el postest, $F_{(2.33)}=5.07, p=.05, \eta_{p}^{2}=.23$, siendo el Grupo $0 \mathrm{~h}$ diferente del Grupo $168 \mathrm{~h}(p<.01)$; ninguna otra comparación resultó significativa $(p>.05)$. En el segundo análisis se comparó por grupo la ejecución entre pruebas, la cual fue significativamente diferente $(p<.01)$ en todos

Tabla 1

Diferencias respecto al nivel de azar en la permanencia en el cuadrante reforzado

\begin{tabular}{cccc}
\hline Grupo & $\mathrm{M}(\mathrm{DE})$ & $\mathrm{t}(11)$ & $\mathrm{d}$ \\
\hline $0 \mathrm{~h}$ & $32.85(8.90)$ & $6.94^{* *}$ & 4.18 \\
$48 \mathrm{~h}$ & $25.37(10.37)$ & $3.30^{* *}$ & 1.98 \\
$168 \mathrm{~h}$ & $21.01(7.54)$ & $2.76^{* *}$ & 1.66 \\
\hline
\end{tabular}

Nota. $* * p<.01$, una cola. los casos: Grupo $0 \mathrm{~h}: F_{(1.22)}=29.79, \eta_{p}^{2}=.57$; Grupo $48 \mathrm{~h}$ : $F_{(1.22)}=16.73, \eta_{p}{ }^{2}=.43$; y Grupo $168 \mathrm{~h}: F_{(1.22)}=8.08, \eta_{p}{ }^{2}=.26$. Adicionalmente, las pruebas $t$ mostraron durante el postest una permanencia por encima del nivel del azar (15 s) en el cuadrante reforzado para todos los grupos (véase Tabla 1).

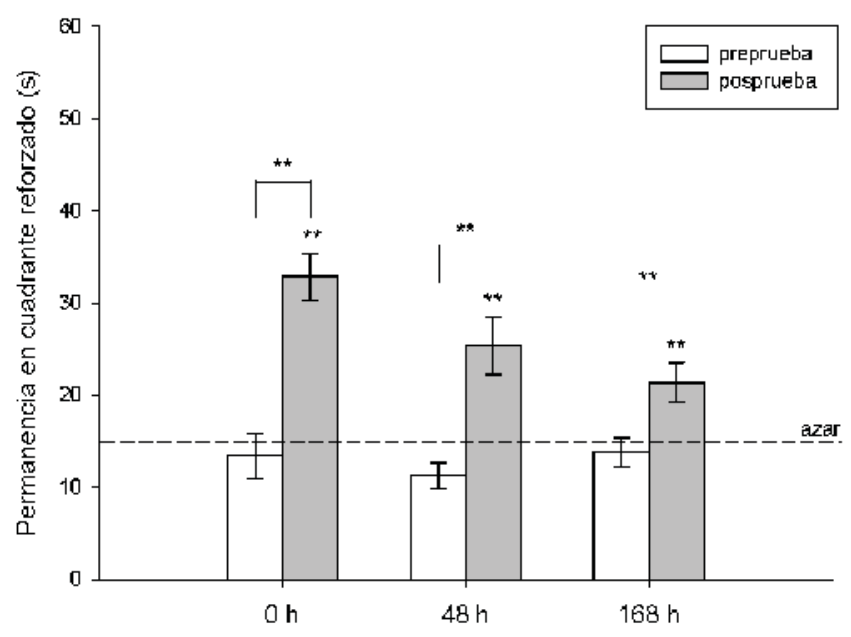

Figura 3. Tiempo de permanencia en el cuadrante reforzado. La barra de error indica el error estándar de la media. $* *=p<.01$.

El ANOVA de 3 grupos vs. 2 pruebas antes descrito fue también empleado para analizar la permanencia en el subcuadrante interno, en el cual la permanencia fue también menor durante el pretest en comparación con el postest para todos los grupos (véase Figura 4). Este análisis reveló un efecto principal $(p<.01)$ para cada factor, es decir, $F_{(2.66)}=6.23, \eta_{p}^{2}=.15$ para los grupos; y $F_{(1.66)}=77.39$, $\eta_{p}^{2}=.54$ para las pruebas, siendo la permanencia del Grupo $168 \mathrm{~h}$ en esta zona diferente a la del Grupo $0 \mathrm{~h}(p<.01)$ y a la del Grupo $48 \mathrm{~h}(p=.05)$. Ninguna otra comparación resultó significativa $(p>.05)$, salvo para el Grupo $168 \mathrm{~h}$, donde la permanencia durante el postest en esta misma zona rebasó el nivel del azar, el cual fue de 8.97 s (véase Tabla 2).

Tabla 2

Diferencias respecto al nivel de azar en la permanencia en el subcuadrante reforzado

\begin{tabular}{llll}
\hline Grupo & $\mathrm{M}(\mathrm{DE})$ & $\mathrm{t}(11)$ & $\mathrm{d}$ \\
\hline $0 \mathrm{~h}$ & $19.29(5.81)$ & $6.16^{* *}$ & 3.70 \\
$48 \mathrm{~h}$ & $15.95(8.00)$ & $3.02^{* *}$ & 1.82 \\
$168 \mathrm{~h}$ & $11.00(5.76)$ & 1.22 n. s. & - \\
\hline
\end{tabular}

Nota. ${ }^{* *} p<.01$, una cola; n. $\mathrm{s} .=$ no significativo. 


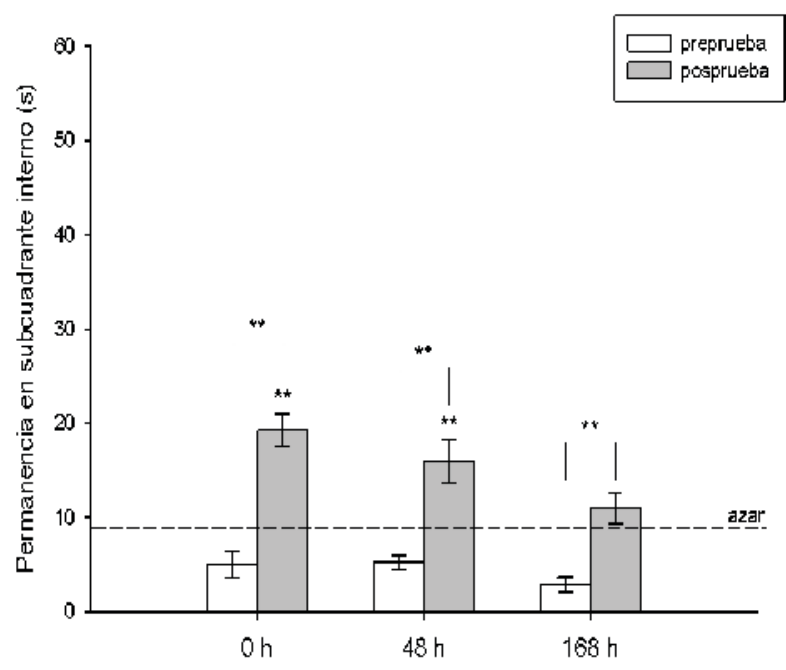

Figura 4. Tiempo de permanencia en el subcuadrante reforzado. La barra de error indica el error estándar de la media. $* *=p<.01$.

El IP se presenta en la Figura 5. Un ANOVA unifactorial mostró diferencias entre los grupos, $F_{(2.33)}=3.64, p<.05$, $\eta_{p}{ }^{2}=.18$, siendo el Grupo $48 \mathrm{~h}$ distinto del Grupo $168 \mathrm{~h}$ $(p<.05)$. Ninguna otra comparación resultó significativa $(p>.05)$. Adicionalmente, las pruebas $t$ mostraron que el punto de indiferencia para el IP (.5) fue rebasado solo por el Grupo 0 h: $t(11)=2.92,(d=1.76)$; y el Grupo $48 \mathrm{~h}$ : $t(11)=4.41(d=2.65), p<.01$.

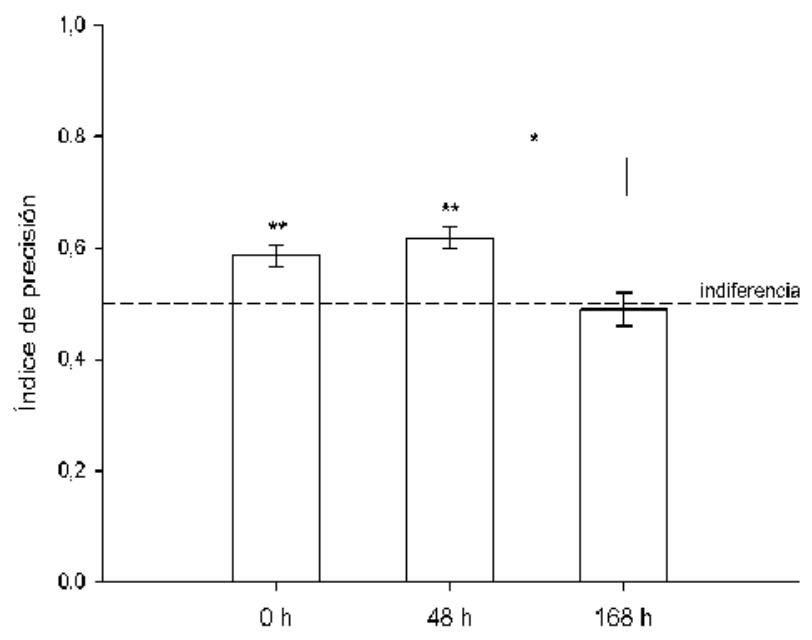

Figura 5. Índice de precisión en la conducta de búsqueda de la meta. La barra de error indica el error estándar de la media. $*=p<.05, * *=p<.01$.

Finalmente, la distancia de nado recorrida en el LVA $y$ en el cuadrante reforzado fue mayor durante el postest respecto de la registrada para el pretest en las mismas zonas. Esto se muestra en la Figura 6 y Figura 7, respectivamente. Para el primer caso, esto se confirmó mediante un ANOVA

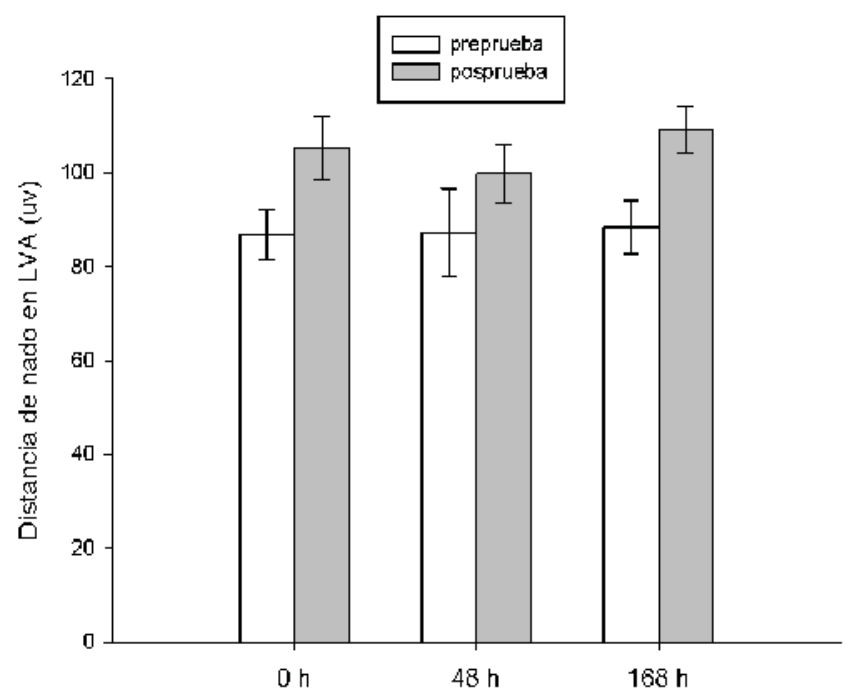

Figura 6. Distancia de nado en unidades virtuales realizada en la totalidad del LVA. La barra de error indica el error estándar de la media.

de 3 grupos vs. 2 pruebas, el cual reveló un efecto principal para el último factor, $F_{(1.66)}=11.70, p<.01, \eta_{p}{ }^{2}=.15$. Para el segundo caso, el análisis antes descrito mostró un efecto principal para cada factor y su interacción, a decir: (a) grupos: $F_{(2.66)}=12.65, p<.01, \eta_{p}{ }^{2}=.27$; (b) pruebas: $F_{(1.66)}=59.55$, $p<.01, \eta_{p}{ }^{2}=.47$; y (c) grupos vs. pruebas: $F_{(2.66)}=9.15$, $p<.01, \eta_{p}^{2}=.21$. Por otra parte, para el análisis de la interacción se condujeron inicialmente dos ANOVA unifactoriales, a fin de comparar entre grupos el desempeño durante cada prueba. Aunque no se detectaron diferencias para el pretest $(p>.05)$, sí las hubo para el postest, donde $F_{(2.33)}=15.48$, $p=.01, \eta_{p}{ }^{2}=.48$, siendo el Grupo $0 \mathrm{~h}$ diferente del Grupo $48 \mathrm{~h}(p<.05)$ y del Grupo $168 \mathrm{~h}(p<.01)$, y el Grupo $48 \mathrm{~h}$

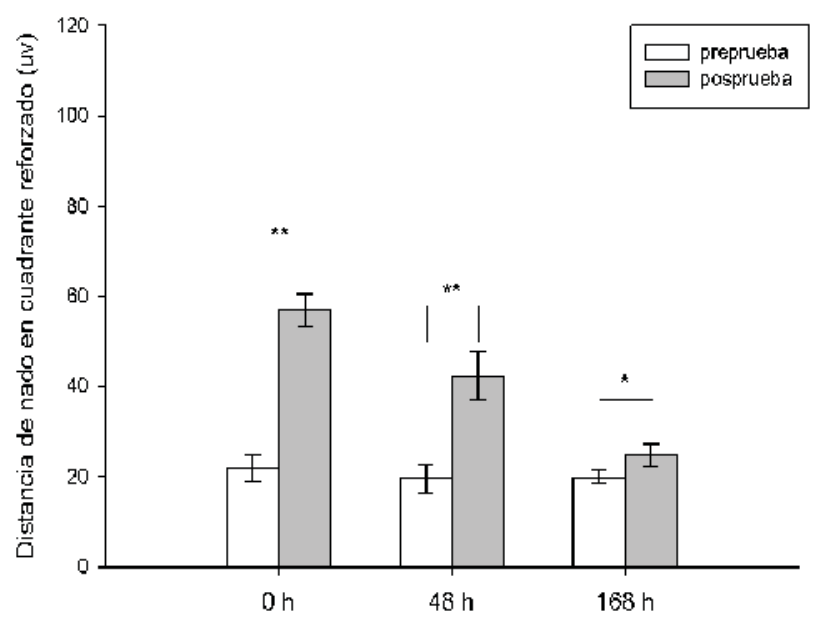

Figura 7. Distancia de nado en unidades virtuales realizada en el cuadrante reforzado. La barra de error indica el error estándar de la media. $*=p<.05, * *=p<.01$. 
diferente del Grupo 168 h $(p=.01)$. Posteriormente, se realizaron tres ANOVA unifactoriales para comparar por grupo la ejecución entre pruebas. En todos los casos se reportaron diferencias significativas, $F_{(1.22)}=55.32\left(\eta_{p}^{2}=.48\right)$, $13.56\left(\eta_{p}{ }^{2}=.38\right), p<.01$, para el Grupo $0 \mathrm{~h}$ y el Grupo $48 \mathrm{~h}$, respectivamente, donde el Grupo $168 \mathrm{~h}$ obtuvo un $F_{(1.22)}=4.59, p<.05, \eta_{p}^{2}=.17$.

\section{DISCUSIÓN}

El objetivo de esta investigación fue describir el efecto del paso del tiempo en la memoria espacial en humanos. Los datos obtenidos demuestran que después de su adquisición, la memoria espacial se retuvo sin cambios significativos hasta por un lapso de $48 \mathrm{~h}$. Cuando el lapso fue de $168 \mathrm{~h}$, esta se modificó en su contenido, lo cual se reflejó en la búsqueda menos persistente y precisa de la meta en el sitio en donde previamente se había encontrado.

La ejecución de los participantes durante las primeras fases del estudio (práctica y preentrenamiento) demostró la habilidad motora y visual pertinente para desplazarse adecuadamente en el LVA. El desconocimiento inicial de la ubicación de la plataforma se hizo evidente por la falta de preferencia por algún cuadrante de entorno, registrada durante el pretest, así como por la prolongada latencia de escape en el primer ensayo de la fase de entrenamiento. Sin embargo, la reducción en esta variable sobre el curso de los ensayos de entrenamiento restantes demostró el establecimiento de la memoria espacial.

Lo anterior se confirmó con la ejecución del Grupo $0 \mathrm{~h}$ durante el postest, pues se detectó la preferencia por el cuadrante reforzado. Tales resultados son consistentes con los obtenidos en otros estudios que emplearon el laberinto de agua tradicional con roedores (Baldi et al., 2005; Blokland et al., 2004; Clark et al., 2005; Morris, 1984) y el virtual con humanos (Astur et al., 1998; Goodrich-Hunsaker et al., 2009; Hamilton et al., 2002; Jacobs et al., 1997; Kolarik et al., 2016; Schoenfeld et al., 2014).

La preferencia por cuadrante reforzado durante el postest, registrada en todos los grupos, indica que la memoria espacial en humanos se retiene y puede ser recuperada hasta siete días posteriores a su adquisición. Esto datos son consistentes con el concepto de memoria a largo plazo (Izquierdo et al., 1999; Roth et al., 2012), y, específicamente, con el de memoria espacial a largo plazo (Demas et al., 1996; Morris, 2013). Un efecto semejante había sido previamente demostrado en roedores (Méndez-Couz et al., 2015), aunque también se encontraron datos opuestos en otro estudio (Carmack et al., 2014).
El análisis detallado de la ejecución durante el postest mostró la ausencia de diferencias en la permanencia en el cuadrante reforzado entre el Grupo $0 \mathrm{~h}$ y el Grupo $48 \mathrm{~h}$. Esto sugiere que el contenido de la memoria espacial a corto y a largo plazo es análogo, si no es que equivalente, al menos por un lapso de hasta $48 \mathrm{~h}$. Sin embargo, la permanencia en el cuadrante reforzado fue mayor en el Grupo $0 \mathrm{~h}$ que la registrada por el Grupo $168 \mathrm{~h}$. Esta relación inversa entre permanencia en el cuadrante reforzado y la prolongada duración del IR es semejante a la obtenida en otros trabajos con roedores (Clark et al., 2005), e implica que, rebasado un lapso de IR, la información contenida en la memoria espacial a corto plazo no es equivalente a la contenida en la memoria espacial a largo plazo. Los datos contrapuestos del Grupo 48 h y del Grupo 168 h, respecto del Grupo 0 $\mathrm{h}$ indican que el momento en que ocurre dicho cambio es posterior a las $48 \mathrm{~h}$. Futuros estudios deberán confirmar dicha afirmación.

El análisis de la permanencia en el subcuadrante interno es útil para esclarecer la forma en que la memoria espacial a largo plazo pudo modificarse en función del paso del tiempo. La ausencia de diferencias en esta variable entre el Grupo 0 h y el Grupo $48 \mathrm{~h}$ refuerza la idea antes mencionada de que el contenido de la memoria espacial a corto y a largo plazo es semejante, al menos en las primeras horas. Sin embargo, ambos grupos fueron diferentes en la variable mencionada respecto del Grupo $168 \mathrm{~h}$. Dado que la permanencia en el subcuadrante interno es un indicador de la precisión de la memoria espacial y de la conducta de búsqueda asociada, es posible suponer que uno de los efectos adversos del paso del tiempo es la pérdida de precisión en este tipo de memoria, un resultado comúnmente reportado en estudios de memoria (Odinot \& Wolters, 2006). A favor de esta interpretación se encuentra el hecho de que el IP del Grupo $168 \mathrm{~h}$ alcanzó el punto de indiferencia, lo que indica que su búsqueda de la plataforma se realizó por igual tanto en el subcuadrante interno como en el área periférica a este, aunque también en el cuadrante reforzado. Lo anterior no ocurrió con los otros grupos, los cuales realizaron una búsqueda más precisa de la meta, mayormente en el área del subcuadrante interno.

La pérdida de precisión en la memoria espacial a largo plazo pudo ser debida a procesos generales de la memoria, específicamente a la retención y recuperación, o a procesos particulares propios a la memoria espacial. Para el primer caso, es viable plantear que el Grupo $0 \mathrm{~h}$ y el Grupo $48 \mathrm{~h}$ adquirieron (fase de entrenamiento), retuvieron (IR) y recuperaron (postest) sin problemas la memoria espacial. En cambio, es posible que el Grupo $168 \mathrm{~h}$ presentara un problema en alguno de los dos últimos procesos, o incluso en ambos. 
De este supuesto se derivan dos explicaciones, la primera de ellas se basa en un efecto de olvido espontáneo (Sara, 2000), ocasionado por la prolongada duración del IR empleado en el Grupo 168 h; de acuerdo a esta explicación, los participantes de dicho grupo habrían sido incapaces de retener la información espacial necesaria para dirigir su conducta de búsqueda hacia el sitio en el que antes se localizó la plataforma oculta; y la segunda explicación propone la ocurrencia de un problema en la recuperación de la información (Sara, 2000), lo que implica que la información acerca de la ubicación de la plataforma se mantuvo, aunque durante el postest los participantes fueron incapaces de recuperarla. Es incluso viable suponer una mezcla de ambas explicaciones.

Por otra parte, una tercera explicación supone la mejora en el recuerdo de la ubicación de la meta en función del paso del tiempo, posiblemente debido a un efecto de consolidación de la memoria espacial (Ferrara et al., 2006). De haber ocurrido esto, los participantes se habrían dirigido al sitio exacto en que la meta se localizó y al no encontrarla allí, tendieron a desplazarse hacia otras áreas del LVA en su búsqueda (Hardt et al., 2009).

Aunque estas tres explicaciones son potencialmente plausibles, los datos actuales no permiten verificar o refutar cualquiera de ellas, por lo que futuros estudios deberán ser diseñados expresamente con ese fin. Para el segundo caso, el referido a procesos particulares propios de la memoria espacial, los datos del Grupo $0 \mathrm{~h}$ y del Grupo $48 \mathrm{~h}$ pueden ser explicados a partir de la TMC (O'Keefe \& Nadel, 1978; Hardt et al., 2009), por suponer que durante la fase de entrenamiento los participantes conformaron una representación mental o mapa cognitivo del LVA.

Esta última representación habría sido útil para desplazarse en forma directa del punto de partida hacia la meta, $y$ así buscar dicha meta en el cuadrante reforzado cuando esta fue omitida durante el postest. No obstante, la ejecución reportada por el Grupo $168 \mathrm{~h}$ no puede ser explicada por esta teoría, ya que de acuerdo a ella el paso del tiempo no tiene ningún efecto sobre el mapa cognitivo. De esta manera, la ejecución de dicho grupo debió haber sido equivalente a la de los grupos restantes.

Una alternativa para dar cuenta de este último resultado sería a partir de los supuestos de la teoría del trazo múltiple (TTM), de Moscovitch et al. (2006), y de la hipótesis de la trasformación de la memoria (HTM), de Winocur, Moscovitch y Sekeres (2007). En términos generales, estas dos aproximaciones proponen que una memoria originalmente dependiente del $\mathrm{HC}$-como lo es la memoria espacial-puede, con el paso del tiempo, transformarse en una memoria independiente de dicha estructura. Con esta transformación, la memoria pierde información específica (por ejemplo, contexto u otros detalles vívidos) del evento meta, conservando solo sus aspectos generales. Una posibilidad es entonces que la memoria espacial de los participantes del Grupo 168 h, inicialmente rica en detalles, se hubiera modificado, perdiendo así información específica acerca de la localización de la plataforma, aunque conservando el recuerdo general de su localización en el LVA. Esto podría explicar la preferencia por el cuadrante reforzado y, al mismo tiempo, un IP equivalente al punto de indiferencia.

El conjunto de resultados obtenido tiene diversas implicaciones. A nivel teórico, y como previamente se ha discutido, revelan la imposibilidad de ser explicados por una de las teorías más relevantes del área: la TMC (O'Keefe \& Nadel, 1978). Esto sugiere la necesidad de integrar en ella, y en modelos relacionados, como el de Burgess, Jackson, Hartley y O'Keefe (2000), mecanismos de retención y recuperación que describan los efectos del paso del tiempo en la memoria espacial. Por otra parte, si bien los planteamientos de la TTM (Moscovitch et al., 2006) y de la HTM (Winocur et al., 2007) son capaces de ofrecer una explicación plausible, se requiere una verificación empírica de esta.

Con respecto a la psicología comparada, los resultados de el presente estudio revelan semejanzas con los obtenidos con roedores al emplear IR semejantes. Esto demuestra la participación de mecanismos de retención de la memoria espacial compartidos entre especies. Tal vez, la implicación más relevante de este estudio sea en un contexto aplicado. Los datos muestran la pertinencia del entorno, el diseño y los indicadores conductuales registrados para estudiar los cambios que ocurren en la memoria espacial en función del paso del tiempo, lo que hace de este LVA y procedimiento una metodología potencialmente útil para su aplicación en poblaciones sanas y clínicas, con lo cual se podría aportar conocimiento novedoso sobre la función típica y patológica de la memoria episódica de humanos en aspectos previamente poco explorados (por ejemplo, precisión) (Kolarik et al., 2016).

Finalmente, es importante señalar que existen algunos aspectos que futuros estudios deberán abordar con el fin de investigar más ampliamente la memoria espacial a largo plazo en humanos. Entre estos se encuentran las diferencias ligadas al sexo en la memoria espacial (Jones $\&$ Healy, 2006) y los efectos diferenciales del uso de un entrenamiento masivo contra un entrenamiento dosificado en el laberinto de agua (Commins, Cunningham, Harvey $\&$ Walsh, 2003).

En conclusión, los datos del presente estudio demuestran la presencia en humanos de una memoria espacial a largo plazo, que es semejante a la memoria espacial a corto plazo al menos hasta $48 \mathrm{~h}$ posteriores a su adquisición, y que después se modifica y ocasiona un cambio en la conducta de búsqueda asociada. 


\section{REFERENCIAS}

Aron. A., \& Aron, E. N. (2001). Estadística para psicólogos. México: Prentice Hall.

Astur, R. S., Ortiz, M. L., \& Sutherland, R. J. (1998). A characterization of performance by men and women in a virtual Morris water task: a large and reliable sex difference. Behavioural Brain Research, 93(1-2), 185-190. doi:http:// dx.doi.org/10.1016/S0166-4328(98)00019-9.

Astur, R. S., Purton, A. J., Zaniewski, M. J., Cimadevilla, J., \& Markus, E. J. (2016). Human sex differences in solving a virtual navigation problem. Behavioural Brain Research, 308, 236-243. doi:10.1016/j.bbr.2016.04.037.

Ayaz, H., Shewokis, P. A., Curtin, A., Izzetoglu, M., Izzetoglu, K., \& Onaral, B. (2011). Using maze suite and functional near infrared spectroscopy to study learning in spatial navigation. Journal of Visualized Experiments, 56, e3443. doi:10.3791/3443.

Baldi, E., Efoudebe, M., Lorenzini, C. A., \& Bucherelli, C. (2005). Spatial navigation in the Morris water maze: working and long lasting reference memories. Neuroscience Letters, 378(3), 176-180. doi:http://dx.doi.org/10.1016/j. neulet.2004.12.029.

Blokland, A., Geraerts, E., \& Been, M. (2004). A detailed analysis of rats' spatial memory in a probe trial of a Morris task. Behavioural Brain Research, 154(1), 71-75. doi:10.1016/j. bbr.2004.01.022.

Burgess, N., Jackson, A., Hartley, T., \& O'Keefe, J. (2000). Predictions derived from modelling the hippocampal role in navigation. Biological Cybernetics, 83(3), 301-312. doi:10.1007/s004220000172.

Burgess, N., Maguire, E. A., \& O'Keefe, J. (2002). The human hippocampus and spatial and episodic memory. Neuron, 35(4), 625-641. doi:http://dx.doi.org/10.1016/S08966273(02)00830-9.

Carmack, S. A., Block, C. L., Howell, K. K., \& Anagnostaras, S. G. (2014). Methylphenidate enhances acquisition and retention of spatial memory. Neuroscience Letters, 567, 4550. doi:10.1016/j.neulet.2014.03.029.

Chalfonte, B. L., \& Johnson, M. K. (1996). Feature memory and binding in young and older adults. Memory \& Cognition, 24(4), 403-416. doi:10.3758/BF03200930.

Chamizo, V. D., Aznar-Casanova, J. A., \& Artigas, A. A. (2003). Human overshadowing in a virtual pool: Simple guidance is a good competitor against locale learning. Learning and Motivation, 34(3), 262-281. doi:https://doi.org/10.1016/ S0023-9690(03)00020-1.

Clark, R. E., Broadbent, N. J., \& Squire, L. R. (2005). Hippocampus and remote spatial memory in rats. Hippocampus, 15(2), 260-272. doi:10.1002/hipo.20056.

Commins, S., Cunningham, L., Harvey, D., \& Walsh, D. (2003). Massed but not spaced training impairs spatial memory. Behavioural Brain Research, 139(1-2), 215-223. doi:http:// dx.doi.org/10.1016/S0166-4328(02)00270-X.
Conejo, N. M., Cimadevilla, J. M., González-Pardo, H., Méndez-Couz, M., \& Arias, J. L. (2013). Hippocampal inactivation with TTX impairs long-term spatial memory retrieval and modifies brain metabolic activity. PloS one, 8, e64749. doi:http://dx.doi.org/10.1371/journal.pone.0064749.

D’Hooge, R., \& De Deyn, P. P. (2001). Applications of the Morris water maze in the study of learning and memory. Brain Research Reviews, 36(1), 60-90. doi:10.1016/S01650173(01)00067-4.

De Winter, J. C. (2013). Using the Student's t-test with extremely small sample sizes. Practical Assessment, Research \& Evaluation, 18(10), 1-12.

Demas, G. E., Nelson, R. J., Krueger, B. K., \& Yarowsky, P. J. (1996). Spatial memory deficits in segmental trisomic Ts65Dn mice. Behavioural Brain Research, 82(1), 85-92. doi:http://dx.doi.org/10.1016/S0166-4328(97)81111-4.

Ferrara, M., Iaria, G., de Gennaro, L., Guariglia, C., Cursio, G., Tempesta, D., \& Bertini, M. (2006). The role of sleep in the consolidation of route learning in humans: A behavioural study. Brain Research Bulletin, 71(1-3), 4-9. doi:http:// dx.doi.org/10.1016/j.brainresbull.2006.07.015.

Florian, C., \& Roullet, P. (2004). Hippocampal CA3-region is crucial for acquisition and memory consolidation in Morris water maze task in mice. Behavioural Brain Research, 154(2), 365-374. doi:http://dx.doi.org/10.1016/j. bbr.2004.03.003.

Goodrich-Hunsaker, N. J., Livingstone, S. A., Skelton, R. W., \& Hopkins, R. O. (2009). Spatial deficits in a virtual water maze in amnesic participants with hippocampal damage. Hippocampus, 20(4), 481-491. doi:10.1002/hipo.20651.

Hamilton, D., Driscoll, I., \& Sutherland, R. J. (2002). Human place learning in a virtual Morris water task: some important constraints on the flexibility of place navigation. Behavioral Brain Research, 129(1-2), 159-170. doi:http:// dx.doi.org/10.1016/S0166-4328(01)00343-6.

Hamilton, D. A., Kodituwakku, P., Sutherland, R. J., \& Savage, D. D. (2003). Children with Fetal Alcohol Syndrome are impaired at place learning but not cued-navigation in a virtual Morris water task. Behavioural Brain Research, 143(1), 85-94. doi:http://dx.doi.org/10.1016/S01664328(03)00028-7.

Hardt, O., Hupbach, A., \& Nadel, L. (2009). Factors moderating blocking in human place learning: The role of task instructions. Learning \& Behavior, 37(1), 42-59. doi:10.3758/ LB.37.1.42.

Izquierdo, I., Medina, J. H., Vianna, M. R. M., Izquierdo, L. A., \& Barros, D. M. (1999). Separate mechanisms for short- and long-term memory. Behavioural Brain Research, 103(1), 1-11. doi:http://dx.doi.org/10.1016/S01664328(99)00036-4.

Jacobs, W. J., Laurance, H. E., \& Thomas, K. G. (1997). Place learning in virtual space I: Acquisition, overshadowing, and transfer. Learning and Motivation, 28, 521-541. doi:10.1006/lmot.1997.0977. 
Jones, C. M., \& Healy, S. D. (2006). Differences in cue use and spatial memory in men and women. Proceedings of the Royal Society of London B: Biological Sciences, 273(1598), 2241-2247. doi:10.1098/rspb.2006.3572.

Kelly, D. M., \& Gibson, B. M. (2007). Spatial navigation: Spatial learning in real and virtual environments. Comparative Cognition \& Behavior Reviews, 2, 111-124. doi:10.3819/ccbr.2008.20007.

Khan, A., \& Rayner, G. D. (2003). Robustness to non-normality of common tests for the many-sample location problem. Journal of Applied Mathematics \& Decision Sciences, 7(4), 187-206.

Kolarik, B. S., Shahlaie, K., Hassan, A., Borders, A. A., Kaufman, K. C., Gurkoff, G., ... Ekstrom, A. D. (2016). Impairments in precision, rather than spatial strategy, characterize performance on the virtual Morris Water Maze: A case study. Neuropsychologia, 80, 90-101. doi:10.1016/j. neuropsychologia.2015.11.013.

Maguire, E. A., Nannery, R., \& Spiers, H. J. (2006). Navigation around London by a taxi driver with bilateral hippocampal lesions. Brain, 129(11), 2894-2907. doi:https://doi. org/10.1093/brain/awl286.

Manns, J. R., \& Eichenbaum, H. (2009). A cognitive map for object memory in the hippocampus. Learning \& Memo$r y$, 16(10), 616-624. doi:10.1101/lm.1484509.

Méndez-Couz, M., Conejo, N. M., González-Pardo, H., \& Arias, J. L. (2015). Functional interactions between dentate gyrus, striatum and anterior thalamic nuclei on spatial memory retrieval. Brain research, 1605, 59-69. doi:10.1016/j. brainres.2015.02.005.

Mettke-Hofmann, C., \& Gwinner, E. (2003). Long-term memory for a life on the move. Proceedings of the National Academy of Sciences, 100(10), 5863-5866. doi:10.1073/ pnas. 1037505100 .

Morellini, F. (2013). Spatial memory tasks in rodents: What do they model? Cell Tissue Research, 354(1), 273-286. doi:10.1007/s00441-013-1668-9.

Morris, R. G. M. (1984). Developments of a water-maze procedure for studying spatial learning in the rat. Journal of Neuroscience Methods, 11(1), 47-60. doi:http://dx.doi. org/10.1016/0165-0270(84)90007-4.

Morris, R. (2013). Neurobiology of Learning and Memory. En D. W. Pfaff (Ed.), Neuroscience in the 21st Century (pp. 2173-2211). New York: Springer.

Morris, R. G. M., Garrud, P., Rawlins, J. N. P., \& O'Keefe, J. (1982). Place navigation impaired in rats with hippocampal lesion. Nature, 297(5868), 681-683. doi:10.1038/297681a0.

Morris, R. G., \& Mayes, A. R. (2004). Long-term spatial memory: introduction and guide to the special section. Neuropsychology, 18(3), 403-404. doi:http://dx.doi. org/10.1037/0894-4105.18.3.403.
Moscovitch, M., Nadel, L., Winocur, G., Gilboa, A., \& Rosenbaum, R. S. (2006). The cognitive neuroscience of remote episodic, semantic and spatial memory. Current Opinion in Neurobiology, 16(2), 179-190. doi:http://dx.doi. org/10.1016/j.conb.2006.03.013.

Moser, M. B., \& Moser, E. I. (1998). Distributed encoding and retrieval of spatial memory in the hippocampus. The Journal of Neuroscience, 18(18), 7535-7542.

O'Keefe, J., \& Nadel, L. (1978). The hippocampus as a cognitive map. Oxford: Oxford University Press.

Odinot, G., \& Wolters, G. (2006). Repeated recall, retention interval and the accuracy-confidence relation in eyewitness memory. Applied Cognitive Psychology, 20(7), 973-985. doi:10.1002/acp.1263.

Postma, A., Jager, G., Kessels, R. P., Koppeschaar, H. P., \& Van Honk, J. (2004). Sex differences for selective forms of spatial memory. Brain and Cognition, 54(1), 24-34. doi:10.1016/S0278-2626(03)00238-0.

Rosenbaum, R. S., Cassidy, B. N., \& Herdman, K. A. (2015). Patterns of preserved and impaired spatial memory in a case of developmental amnesia. Frontiers in Human Neuroscience, 9, 196. doi:10.3389/fnhum.2015.00196.

Rosenbaum, R. S., Winocur, G., Grady, C. L., Ziegler, M., \& Moscovitch, M. (2007). Memory for familiar environments learned in the remote past: fMRI studies of healthy people and an amnesic person with extensive bilateral hippocampal lesions. Hippocampus, 17(12), 1241-1251. doi:10.1002/ hipo.20354.

Ross, S. P., Skelton, R. W., \& Mueller, S. C. (2006). Gender differences in spatial navigation in virtual space: implications when using virtual environments in instruction and assessment. Virtual Reality, 10(3), 175-184. doi:10.1007/ s10055-006-0041-7.

Roth, T. C., LaDage, L. D., \& Pravosudov, V. V. (2012). Evidence for long-term spatial memory in a parid. Animal Cognition, 15(2), 149-154. doi:10.1007/s10071-011-0440-3.

Ruetti, E., Justel, N., \& Bentosela, M. (2009). Perspectivas clásicas y contemporáneas acerca de la memoria. Suma Psicológica, 16(1), 65-83.

Sara, S. J. (2000). Retrieval and reconsolidation: Toward a neurobiology of remembering. Learning \& Memory, 7(2), 7384. doi:10.1101/lm.7.2.73.

Shiflett, M. W., Tomaszycki, M. L., Rankin, A. Z., \& DeVoogd, T. J. (2004). Long-term memory for spatial locations in a food-storing bird (Poecile atricapilla) requires activation of NMDA receptors in the hippocampal formation during learning. Behavioral Neuroscience, 118(1), 121-130. doi:http://doi.apa.org/getdoi.cfm?d oi $=10.1037 / 0735-7044.118 .1 .12$.

Schoenfeld, R., Foreman, N., \& Leplow, B. (2014). Ageing and spatial reversal learning in humans: Findings from a virtual water maze. Behavioural Brain Research, 270, 47-55. doi:10.1016/j.bbr.2014.04.036. 
Sociedad Mexicana de Psicología. (2010). Código ético del psicólogo. México: Trillas.

Spieker, E. A., Astur, R. S., West, J. T., Griego, J. A., \& Rowland, L. M. (2012). Spatial memory deficits in a virtual reality eight-arm radial maze in schizophrenia. Schizophrenia Research, 135(1-3), 84-89. doi:10.1016/j.schres.2011.11 .014 .

Spreng, M., Rossier, J., \& Schenk, F. (2002). Spaced training facilitates long-term retention of place navigation in adult but not in adolescent rats. Behavioural Brain Research, 128(1), 103-108. doi:http://dx.doi.org/10.1016/S01664328(01)00266-2.

Squire, L. R. (2004). Memory systems of the brain: A brief history and current perspective. Neurobiology of Learning and Memory, 82(3), 171-177. doi:10.1016/j. $\mathrm{nlm} .2004 .06 .005$.

Teng, E., \& Squire, L. R. (1999). Memory for places learned long ago is intact after hippocampal damage. Nature, 400(6745), 675-677. doi:10.1038/23276.

Tramoni, E., Felician, O., Barbeau, E. J., Guedj, E., Guye, M., Bartolomei, F., \& Ceccaldi, M. (2011). Long-term consolidation of declarative memory: insight from temporal lobe epilepsy. Brain, 134(3), 816-831. doi:10.1093/brain/ awr002.
Vorhees, C. V., \& Williams, M. T. (2014). Assessing spatial learning and memory in rodents. ILAR Journal, 55(2), 310332. doi:https://doi.org/10.1093/ilar/ilu013.

Wilkie, D. M., \& Willson, R. J. (1989). Pigeons' (Columba livia) spatial reference memory is stable over long retention intervals. Bulletin of the Psychonomic Society, 27(3), 271-273. doi:10.3758/BF03334604.

Winocur, G., Moscovitch, M., \& Sekeres, M. (2007). Memory consolidation or transformation: context manipulation and hippocampal representations of memory. Nature Neuroscience, 10, 555-557. doi:10.1038/nn1880.

Woolley, D. G., Laeremans, A., Gantois, I., Mantini, D., Vermaercke, B., Op de Beeck, ... D'Hooge, R. (2013). Homologous involvement of striatum and prefrontal cortex in rodent and human water maze learning. Proceedings of the National Academy of Sciences, 110(8), 3131-3136. doi: doi/10.1073/pnas.1217832110.

World Medical Association Declaration of Helsinki. (2008). Ethical principles for medical research involving human subjects. In World Medical Association. Recuperado de http://www.wma.net/en/30publications/10policies/b3/index.html 